\title{
Asymmetric orientational writing dependence on polarization and direction in glass with femtosecond laser irradiation
}

\section{Bertrand Poumellec, ${ }^{2, *}$ Matthieu Lancry, ${ }^{2}$ Chaxing Fan, ${ }^{1,2}$ Rudy Desmarchelier, ${ }^{2}$ Huidan Zeng, ${ }^{1, *}$ Bernard Bouguignon, ${ }^{3}$ and Guorong Chen 1}

${ }^{1}$ Key Laboratory for Ultrafine Materials of Ministry of Education, School of Materials Science and Engineering, East China University of Science and Technology, Shanghai 200237, China

${ }^{2}$ Institut de Chimie Moléculaire et des Matériaux d'Orsay (ICMMO), UMR CNRS-PSUD 8182, Bâtiment 410, and

3 Institut des Sciences Moléculaires d'Orsay, CNRS-PSUD UPR 3361, Bâtiment 350, Université de Paris Sud, 91405 Orsay, France.

We observed an asymmetry with the orientation of the laser scanning firstly in 2003 in pure silica [1] measuring the strain induced by the laser irradiation. P. Kazansky showed that also in the micro-structure of the laser trace in pure silica [2]. From that date, we have found such a phenomenon in several glasses and several properties. Because a strong birefringence, photo-induced by the femtosecond laser irradiation, has been achieved in $32.5 \mathrm{Li}_{2} \mathrm{O}-$ $27.5 \mathrm{Nb}_{2} \mathrm{O}_{5}-40 \mathrm{SiO}_{2}$ glass, we studied this effect in this glass, taking advantage that it is out of the nanostructures of low index pointed out by Kazansky et al [2] at the basis of form birefringence.

The sensitivity of the birefringence to the orientation of the laser movement versus the laboratory reference (i.e. difference between rightward and leftward or between upward and downward) is confirmed. We show in addition that this asymmetric orientational sensitivity is dependent on the directions of laser polarization and of the laser scanning (i.e. when the direction are in the plan of the laser compressor or perpendicular to it). These observations are not explained by the model proposed by Kazansky [3].

The figure 1 shows the configurations that we investigated.

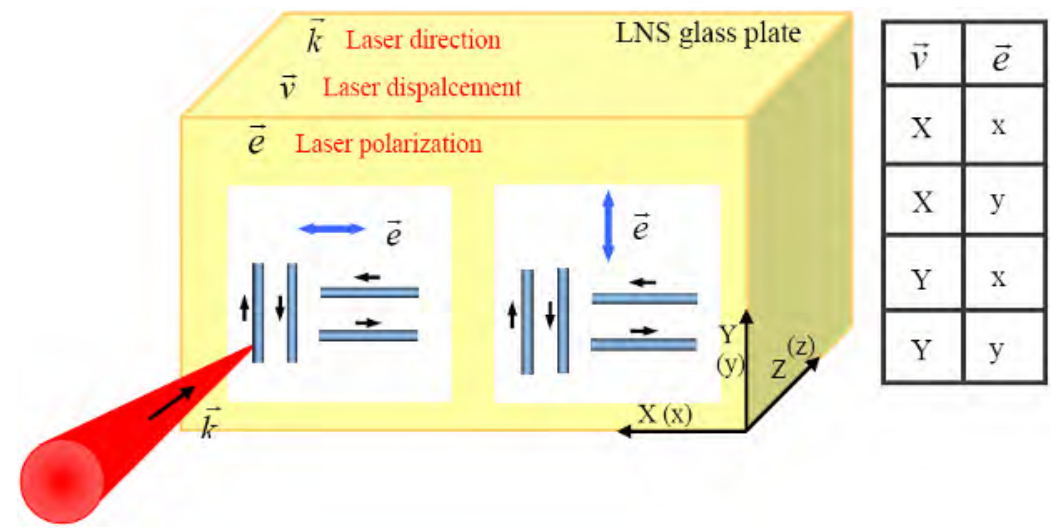

Figure 1 Experimental scheme of femtosecond laser writing geometry in LNS glass. $\vec{v}:$ laser displacement; $\vec{e}$ : laser polarization. The four configurations Xx, Xy, Yx, Yy were investigated. 
Workshop : Progress in Ultrafast Laser Modifications of Materials, Cargese Corsica, France, 14-19 April 2013
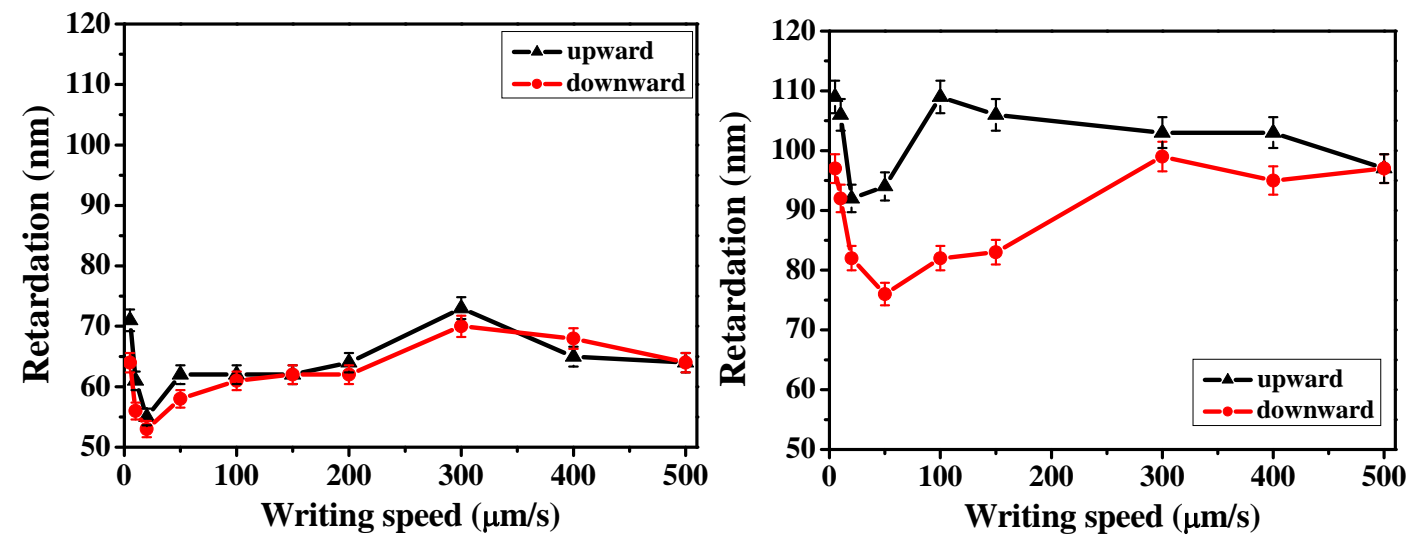

Figure 2: Plot of the retardation with respect to writing velocity along $+Y$ axis (black dots) and $-Y$ axis (red dots). a) Yy writing; b) Yx writing. Fixed pulse energy = $2.3 \mu \mathrm{J} /$ pulse.
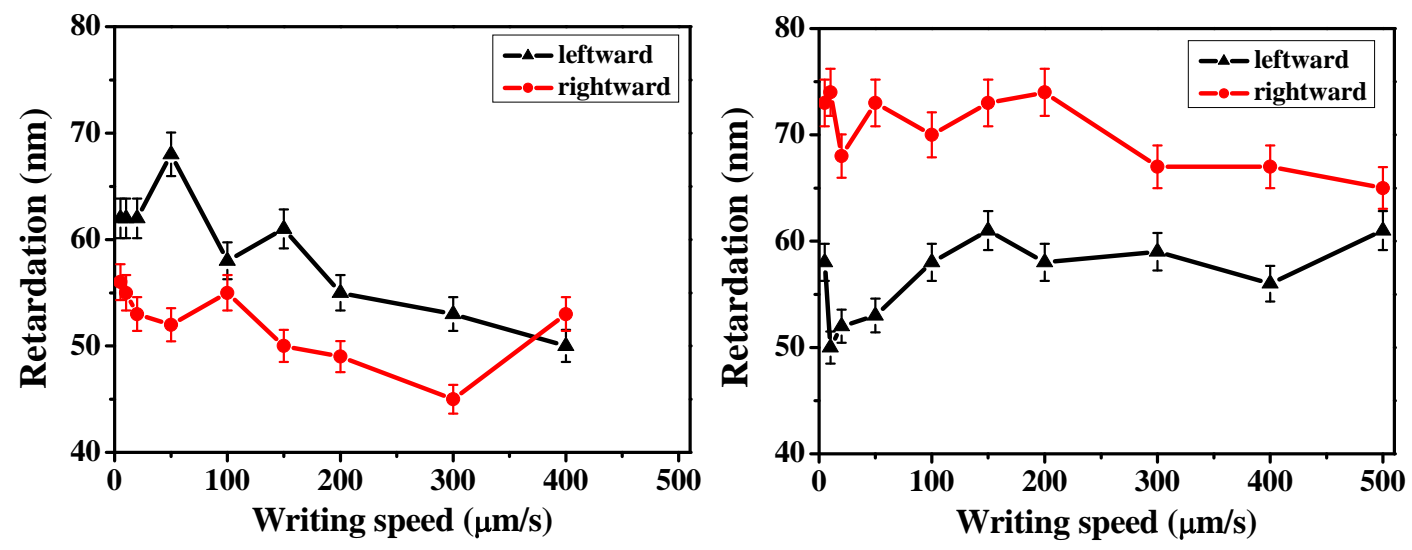

Figure 3: Plot of the retardation with respect to writing velocity in left-to-right $(+X)$ direction (black triangles) and right-to-left (-X) direction (red dots): a) parallel polarization $\mathrm{Xx}$; b) perpendicular polarization Xy. Fixed pulse energy $=2.3 \mu \mathrm{J} /$ pulse.

We revealed that high photo-induced birefringence as much as $2 \times 10^{-3}$ can be achieved in this kind of glasses as long as large negative index change. We suggest that the change of index arises from the increase of fictive temperature induced by femtosecond laser irradiation. It is the first time, we have completed information about the configurational dependence of the asymmetry orientational writing. Contrarily to what it was shown before by Kazansky et al. [2], the largest modifications (see Fig. 2 and Fig. 3) are not in the laser pulse compression plan but depend on the physical quantity observed. For the birefringence, here, we detected no asymmetrical effect only when both writing and polarization are perpendicular to the compression plan of the laser.

\section{Acknowledgments}

This work has been done in the frame of FLAG (Femtosecond Laser Application in Glasses) consortium project with the support of several organizations: the Agence Nationale pour la Recherche (ANR-09BLAN-0172-01), the RTRA Triangle de la Physique (2008-056T), the FP7-PEOPLE-IIF FemtoNano 908582, Shanghai Leading Academic Discipline Project (No. B502), Shanghai Key Laboratory Project (08DZ2230500), the National Natural Science Foundation of China (NSFC51072052), the Natural Science Foundation of Shanghai (12ZR1407600), and the Fundamental Research Funds for the Central Universities (WD1014035).

\section{References}

[1] B. Poumellec, L. Sudrie, M. Franco, B. Prade, and A. Mysyrowicz, Opt. Express 11, 1070 (2003).

[2] P. G. Kazansky, W. Yang, E. Bricchi, J. Bovatsek, A. Arai, Y. Shimotsuma, K. Miura, and K. Hirao, Applied Physics Letters 90, 151120 (2007).

[3] P. G. Kazansky, Y. Shimotsuma, M. Sakakura, M. Beresna, M. Gecevičius, Y. Svirko, S. Akturk, J. Qiu, K. Miura, and K. Hirao, Opt. Express 19, 20657 (2011). 